\title{
miR-125b and miR-100 Are Predictive Biomarkers of Response to Induction Chemotherapy in Osteosarcoma
}

\author{
Daisuke Kubota, ${ }^{1,2}$ Nobuyoshi Kosaka, ${ }^{3}$ Tomohiro Fujiwara, ${ }^{3}$ \\ Akihiko Yoshida, ${ }^{4}$ Yasuhito Arai, ${ }^{5}$ Zhiwei Qiao, ${ }^{1}$ Fumitaka Takeshita, ${ }^{6}$ \\ Takahiro Ochiya, ${ }^{3}$ Akira Kawai, ${ }^{5}$ and Tadashi Kondo ${ }^{1}$ \\ ${ }^{1}$ Division of Rare Cancer Research, National Cancer Center Research Institute, 5-1-1 Tsukiji, Chuo-ku, Tokyo 104-0045, Japan \\ ${ }^{2}$ Division of Musculoskeletal Oncology, National Cancer Center Hospital, 5-1-1 Tsukiji, Chuo-ku, Tokyo 104-0045, Japan \\ ${ }^{3}$ Division of Molecular and Cellular Medicine, National Cancer Center Research Institute, 5-1-1 Tsukiji, \\ Chuo-ku, Tokyo 104-0045, Japan \\ ${ }^{4}$ Pathology and Clinical Laboratory Division, National Cancer Center Hospital, 5-1-1 Tsukiji, Chuo-ku, Tokyo 104-0045, Japan \\ ${ }^{5}$ Division of Cancer Genomics, National Cancer Center Research Institute, 5-1-1 Tsukiji, Chuo-ku, Tokyo 104-0045, Japan \\ ${ }^{6}$ Department of Functional Analysis, National Cancer Center Research Institute, 5-1-1 Tsukiji, Chuo-ku, Tokyo 104-0045, Japan
}

Correspondence should be addressed to Tadashi Kondo; proteomebioinformatics@gmail.com

Received 11 July 2016; Revised 4 October 2016; Accepted 18 October 2016

Academic Editor: Valerae O. Lewis

Copyright (C) 2016 Daisuke Kubota et al. This is an open access article distributed under the Creative Commons Attribution License, which permits unrestricted use, distribution, and reproduction in any medium, provided the original work is properly cited.

Osteosarcoma is the most common primary malignancy in bone. Patients who respond poorly to induction chemotherapy are at higher risk of adverse prognosis. The molecular basis for such poor prognosis remains unclear. We investigated miRNA expression in eight open biopsy samples to identify miRNAs predictive of response to induction chemotherapy and thus maybe used for risk stratification therapy. The samples were obtained from four patients with inferior necrosis (Huvos I/II) and four patients with superior necrosis (Huvos III/IV) following induction chemotherapy. We found six miRNAs, including miR-125b and miR-100, that were differentially expressed $>2$-fold $(p<0.05)$ in patients who respond poorly to treatment. The association between poor prognosis and the abundance of miR-125b and miR-100 was confirmed by quantitative reverse transcriptase-polymerase chain reaction in 20 additional osteosarcoma patients. Accordingly, overexpression of miR-125b and miR-100 in three osteosarcoma cell lines enhanced cell proliferation, invasiveness, and resistance to chemotherapeutic drugs such as methotrexate, doxorubicin, and cisplatin. In addition, overexpression of miR-125b blocked the ability of these chemotherapy agents to induce apoptosis. As open biopsy is routinely performed to diagnose osteosarcoma, levels of miR-125b and miR-100 in these samples may be used as basis for risk stratification therapy.

\section{Introduction}

Osteosarcoma is the most common primary malignancy in bone and a leading cause of cancer death among children and adolescents $[1,2]$. Cure rates of $15-20 \%$ were achieved in the 1970 s by surgery alone in patients with localized osteosarcoma. These rates dramatically improved to as high as $80 \%$ following the introduction of higher-dose and multiagent chemotherapy regimens and induction chemotherapy $[3,4]$. Induction chemotherapy downstages tumors and facilitates complete resection by inhibiting micrometastatic tumors and decreasing tumor vascularity. Response to induction chemotherapy is histologically evaluated according to the Huvos grading system [5], which is based on the degree of tumor necrosis in surgically resected tissues. Patients with $\geq 90 \%$ tumor necrosis after induction chemotherapy are considered good responders, and all others are deemed to be poor responders [2]. It is noteworthy that histological response to induction chemotherapy is the most reliable prognostic factor, aside from metastasis at time of diagnosis [6-15]. 
Therefore, prediction of response to induction chemotherapy could potentially be used to determine the most appropriate treatment regimen [16].

Although Huvos grading is widely used, it is obtained after chemotherapy and is thus not predictive. On the other hand, clinically useful predictive biomarkers have not been identified, even though osteosarcoma has been extensively characterized. This has prevented effective stratification of patients according to risk of drug resistance and may prevent further innovations in treatment. Therefore, it is imperative to understand the molecular basis of chemoresistance to develop more effective therapies.

Osteosarcoma is genetically heterogeneous among patients, across tumors, and within tumors [17, 18]. Indeed, osteosarcoma karyotypes indicate numerous numerical and structural changes [19]. Therefore, a comprehensive omics approach to survey molecular events at multiple levels may identify novel molecular mechanisms underlying resistance to treatments. Given the complex mechanisms that can contribute to chemoresistance, significant biological insights may yet be uncovered.

Previously, we investigated the proteomic profiles of open biopsy samples obtained from osteosarcoma patients before chemotherapy and identified peroxiredoxin 2 (PRDX2) as a novel predictive biomarker with response to induction chemotherapy with ifosfamide, doxorubicin, and cisplatin [20]. Subsequently, we found PRDX2 to be also predictive of the response to induction chemotherapy with different combinations of drugs, and we characterized its functional significance [21]. As open biopsy is routinely performed to diagnose osteosarcoma, predictive biomarkers that can be measured in samples collected during this procedure may prove to be useful in clinical settings.

microRNAs (miRNAs) are small, noncoding RNA 21-25 nucleotides in length that control growth, development, and differentiation by regulating gene expression posttranscriptionally. The human genome encodes more than 1,000 miRNAs [22] that regulate thousands of human genes [23, 24]. In osteosarcoma, global expression of miRNAs has been examined in relation to onset $[25,26]$, progression $[27,28]$, response to treatments $[29,30]$, and prognosis [31]. However, the clinical significance of these miRNAs has not been definitively established.

In this study, we explored the possibility that expression of miRNAs may have a utility in predicting responsiveness to neoadjuvant chemotherapy in osteosarcoma patients. We analyzed miRNA expression in frozen tissue samples obtained before induction chemotherapy. We found that abundant expression of miR-125b and miR-100 was significantly associated with poor response to chemotherapy. We validated this result using qRT-PCR in an independent sample set and verified the functional significance of these miRNAs by in vitro assays.

\section{Material and Methods}

2.1. Patients and Clinical Information. Frozen clinical specimens, collected by open biopsy before chemotherapy, were retrieved from the National Cancer Center Hospital, Japan.
The samples were obtained from eight patients (Table 1) who were diagnosed between 2009 and 2011 and treated according to the standard treatment protocol with methotrexate, doxorubicin, and cisplatin, which are considered key drugs [32]. Response to preoperative chemotherapy was histologically assessed by a pathologist according to the Huvos grading system [2]; when less than $10 \%$ tumor cells were found to be viable, the patients were defined as responders, and if not, they were considered as nonresponders (chemoresistant). Samples were snap-frozen in liquid nitrogen at the time of collection and stored at $-80^{\circ} \mathrm{C}$.

An independent cohort of 20 patients (Table 2) was used to validate results by qRT-PCR, using formalin-fixed paraffinembedded specimens also collected by open biopsy before chemotherapy. These patients were diagnosed between 1990 and 2008. Nine patients were chemoresistant, and 11 were chemosensitive. All patients were monitored from 0.5 to 11.1 years, with mean 7.7 years. Seven patients have been continuously disease-free, seven died of the disease, and six were living with osteosarcoma.

Written informed consent was obtained from all patients, and the study was approved by the ethics committee of the National Cancer Center.

2.2. RNA Extraction and miRNA and mRNA Array. Frozen tissues were powdered under liquid nitrogen using Multibeads shocker (Yasui Kikai, Osaka, Japan). Total RNA was then extracted using miRNeasy Mini Kit (Qiagen, Venlo, Netherlands). Formalin-fixed paraffin-embedded samples were sectioned at $10 \mu \mathrm{m}$, and total RNA was extracted from several of such slices using the miRNeasy FFPE Kit (Qiagen).

miRNA expression profiles of frozen samples were obtained by hybridizing total RNA to the Agilent human miRNA Microarray V3 (021827, $8 \times 15 \mathrm{~K}$, v12.0, Agilent Technologies, Santa Clara, CA), following the manufacturer's instructions. Hybridized microarrays were scanned with a DNA microarray scanner (Agilent G2565BA) with default protocols and settings. miRNA expression was analyzed in GeneSpring GX software (Agilent Technologies). miRNAs were considered differentially expressed when expression increased or decreased $>1.5$-fold, with $p<0.05$ in an unpaired $t$-test.

mRNA expression profiles of osteosarcoma cells were obtained by hybridizing total RNA to SurePrint G3 Human GE microarray $(8 \times 60 \mathrm{~K}$, Ver3.0, Agilent Technologies, Santa Clara, CA), following the manufacturer's instructions. The DNA microarray data were analyzed by Bioconductor agilp package (http://bioconductor.org/packages/agilp/). The probes were selected, when their intensity was considerably (more than 1.5-fold change) different between two samples.

2.3. Validation of $m i R-100$ and $m i R-125 b$ in Independent Samples. miR-100 and miR-125b were amplified by quantitative real-time reverse transcriptase-polymerase chain reaction (qRT-PCR) from formalin-fixed paraffin-embedded samples. First, cDNA was synthesized from $10 \mathrm{ng}$ total RNA using TaqMan MicroRNA hsa-miR-100 and hsa-miR-125b (Applied Biosystems) and TaqMan MicroRNA Reverse Transcription 
TABLE 1: Clinical and pathological data of osteosarcoma patients for global miRNA expression study.

\begin{tabular}{lcccccc}
\hline Case number & Gender & Age & Location & Histologic type & Neoadjuvant chemotherapy $^{\text {Viable cells }(\%)^{\mathrm{A}}}$ \\
\hline Case 1 & Female & 11 & Tibia & Osteoblastic & MTX + DOX + CDDP & $0 \%$ \\
Case 2 & Male & 18 & Tibia & Osteoblastic & MTX + DOX + CDDP & 9\% \\
Case 3 & Male & 15 & Tibia & Osteoblastic & MTX + DOX + CDDP & $0 \%$ \\
Case 4 & Female & 13 & Femur & Osteoblastic & MTX + DOX + CDDP & $0 \%$ \\
Case 5 & Female & 10 & Femur & Osteoblastic & MTX + DOX + CDDP & $70 \%$ \\
Case 6 & Male & 25 & Tibia & Osteoblastic & MTX + DOX + CDDP & $40 \%$ \\
Case 7 & Male & 12 & Femur & Osteoblastic & MTX + DOX + CDDP & $20 \%$ \\
Case 8 & Female & 16 & Femur & Osteoblastic & MTX + DOX + CDDP & $22 \%$ \\
\hline
\end{tabular}

A: probability of viable cells was pathologically evaluated by surgical specimen.

TABLE 2: Clinical and pathological data of osteosarcoma patients for validation study.

\begin{tabular}{|c|c|c|c|c|c|c|c|}
\hline Case number & Gender & Age & Location & Histologic type & Neoadjuvant chemotherapy & Viable cells $(\%)^{\mathrm{A}}$ & Prognosis $^{\mathrm{C}}$ \\
\hline Number 1 & Male & 19 & Tibia & Osteoblastic & $\mathrm{MTX}+\mathrm{DOX}+\mathrm{CDDP}$ & $0-10 \%$ & NED \\
\hline Number 2 & Female & 9 & Femur & Osteoblastic & $\mathrm{MTX}+\mathrm{DOX}+\mathrm{CDDP}$ & $0 \%$ & $\mathrm{CDF}$ \\
\hline Number 3 & Female & 9 & Tibia & Chondroblastic & $\mathrm{MTX}+\mathrm{DOX}+\mathrm{CDDP}$ & $0-10 \%$ & DOD \\
\hline Number 4 & Female & 14 & Femur & Osteoblastic & $\mathrm{MTX}+\mathrm{DOX}+\mathrm{CDDP}$ & 0 & $\mathrm{CDF}$ \\
\hline Number 5 & Female & 15 & Femur & Osteoblastic & $\mathrm{MTX}+\mathrm{DOX}+\mathrm{CDDP}$ & $0-10 \%$ & DOD \\
\hline Number 6 & Female & 9 & Tibia & Osteoblastic & $\mathrm{MTX}+\mathrm{DOX}+\mathrm{CDDP}$ & $0 \%$ & NED \\
\hline Number 7 & Male & 17 & Femur & Osteoblastic & $\mathrm{MTX}+\mathrm{DOX}+\mathrm{CDDP}$ & $0-10 \%$ & $\mathrm{CDF}$ \\
\hline Number 8 & Female & 17 & Tibia & Osteoblastic & $\mathrm{MTX}+\mathrm{DOX}+\mathrm{CDDP}$ & $1 \%$ & $\mathrm{CDF}$ \\
\hline Number 9 & Female & 11 & Femur & Chondroblastic & $\mathrm{MTX}+\mathrm{DOX}+\mathrm{CDDP}$ & $7 \%$ & $\mathrm{CDF}$ \\
\hline Number 10 & Male & 13 & Femur & Osteoblastic & $\mathrm{MTX}+\mathrm{DOX}+\mathrm{CDDP}$ & $0-10 \%$ & DOD \\
\hline Number 11 & Male & 16 & Femur & Chondroblastic & $\mathrm{MTX}+\mathrm{DOX}+\mathrm{CDDP}$ & $0-10 \%$ & DOD \\
\hline Number 12 & Female & 13 & Tibia & Osteoblastic & $\mathrm{MTX}+\mathrm{DOX}+\mathrm{CDDP}$ & $60-70 \%$ & DOD \\
\hline Number 13 & Male & 22 & Femur & Osteoblastic & $\mathrm{MTX}+\mathrm{DOX}+\mathrm{CDDP}$ & $30 \%$ & DOD \\
\hline Number 14 & Male & 18 & Femur & Osteoblastic & $\mathrm{MTX}+\mathrm{DOX}+\mathrm{CDDP}$ & $60-70 \%$ & NED \\
\hline Number 15 & Male & 14 & Tibia & Osteoblastic & $\mathrm{MTX}+\mathrm{DOX}+\mathrm{CDDP}$ & $50-60 \%$ & $\mathrm{CDF}$ \\
\hline Number 16 & Male & 13 & Femur & Osteoblastic & $\mathrm{MTX}+\mathrm{DOX}+\mathrm{CDDP}$ & $30-40 \%$ & AWD \\
\hline Number 17 & Male & 9 & Femur & Chondroblastic & $\mathrm{MTX}+\mathrm{DOX}+\mathrm{CDDP}$ & $30-40 \%$ & NED \\
\hline Number 18 & Female & 10 & Femur & Chondroblastic & $\mathrm{MTX}+\mathrm{DOX}+\mathrm{CDDP}$ & $70 \%$ & $\mathrm{CDF}$ \\
\hline Number 19 & Male & 17 & Ilium & Fibroblastic & $\mathrm{MTX}+\mathrm{DOX}+\mathrm{CDDP}$ & Clinically poor ${ }^{\mathrm{B}}$ & DOD \\
\hline Number 20 & Male & 15 & Tibia & Osteoblastic & MTX + DOX + CDDP & $50-60 \%$ & NED \\
\hline
\end{tabular}

A: Probability of viable cells was pathologically evaluated by surgical specimen. B: patients with disease progression in diagnostic imaging by computed tomography and magnetic resource imaging. C: CDF: continuously disease-free, DOD: died of disease, NED: no evidence of disease, and AWD: alive with disease.

Kit (Applied Biosystems). Target miRNAs were amplified over 45 cycles of denaturation at $95^{\circ} \mathrm{C}$ for $10 \mathrm{sec}$, annealing at $60^{\circ} \mathrm{C}$ for $10 \mathrm{sec}$, and extension at $65^{\circ} \mathrm{C}$ for $10 \mathrm{sec}$, following initial denaturation at $95^{\circ} \mathrm{C}$ for $10 \mathrm{~min}$. PCR was performed in triplicate in 96-well plates using the 7300 Real-Time PCR System (Applied Biosystems), and miRNA expression was normalized to small nuclear RNA RNU6B (Applied Biosystems).

2.4. Cell Culture and Transfection. Differentially expressed miRNAs were functionally characterized in osteosarcoma cell lines MNNG-HOS, 143B, and MG63, American Type Culture Collection. Briefly, cells were cultured at $37^{\circ} \mathrm{C}$ in a humidified $5 \% \mathrm{CO}_{2}$ chamber using DMEM supplemented with $10 \%$ fetal bovine serum, $1 \mathrm{mmol} / \mathrm{L}$ sodium pyruvate, nonessential amino acids, and $2 \mathrm{mmol} / \mathrm{L}$ glutamine. Cells were then transfected with $25 \mathrm{nM}$ negative control miRNA (Cosmo Bio, Tokyo, Japan), miR-100 (Bonac Corporation, Fukuoka, Japan), and miR-125 (Bonac Corporation, Fukuoka, Japan) using DharmaFECT transfection reagents (Thermo Scientific, Tokyo, Japan), following the manufacturer's protocol.

2.5. Cell Proliferation Assay. Cell proliferation was measured using a standard MTS assay. In brief, cells were seeded at 3 $\times 10^{3}$ cells/well in a 96-well plate containing $100 \mu \mathrm{L}$ culture medium, grown overnight, and transfected with miRNAs as described or incubated in conditioned medium. The media in transfected cells were replaced with fresh medium A $24 \mathrm{~h}$ after transfection. Cell viability was determined $3 \mathrm{~d}$ thereafter by Cell Counting Kit-8 (Dojindo, Kumamoto, Japan) according to the manufacturer's protocol. In this assay, WST- 8 is 
reduced by dehydrogenase activity in viable cells to yield a formazan dye, which is soluble in tissue culture media, and assayed by absorbance at $450 \mathrm{~nm}$ [33]. The amount of formazan dye was produced in directly proportional to the number of viable cells. Three wells were examined for each treatment, and experiments were independently repeated three times. Results are reported as mean \pm SD. Statistical significance was tested by $t$-test.

2.6. Cell Invasion Assay. Cell invasion was evaluated using the BD BioCoat ${ }^{\mathrm{Tm}}$ Invasion Chamber (BD Bioscience), following the manufacturer's protocol. In brief, cells transfected with miR-125b or miR-100 for $24 \mathrm{~h}$ were seeded onto the membrane in the upper chamber of the transwell at $5 \times 10^{5}$ cells in $500 \mu \mathrm{L}$ serum-free medium. The medium in the lower chamber contained $10 \%$ fetal calf serum as source of chemoattractants. Cells that passed through the Matrigel-coated membrane were stained with Diff-Quick (Sysmex, Kobe, Japan) and counted. Experiments were performed three times independently, and statistical significance was determined by $t$-test.

2.7. Apoptosis Assay. Cells were transfected as described with $25 \mathrm{nM}$ negative control miRNA, miR-100, and miR-125b and treated $24 \mathrm{~h}$ thereafter with methotrexate for $72 \mathrm{~h}$, doxorubicin for $48 \mathrm{~h}$, and cisplatin for $48 \mathrm{~h}$. Proteins were extracted, and expression of apoptosis-associated proteins was measured by western blotting.

2.8. Protein Extraction and Western Blotting. Frozen tissues were powdered under liquid nitrogen with the Multi-beads shocker and resuspended in $6 \mathrm{~mol} / \mathrm{L}$ urea, $2 \mathrm{~mol} / \mathrm{L}$ thiourea, $3 \%$ CHAPS, and $1 \%$ Triton X-100. The supernatant was then cleared by centrifugation at 15,000 rpm for $30 \mathrm{~min}$. Cultured cells were washed with PBS, fixed with $10 \%$ TCA for $30 \mathrm{~min}$, and lysed in the same buffer for $30 \mathrm{~min}$. The supernatant was collected after centrifugation for $30 \mathrm{~min}$.

Proteins were separated on SDS-polyacrylamide gels and transferred to a nitrocellulose membrane (Bio-Rad). After blocking for $1 \mathrm{~h}$ in Tris-buffered saline and Tween 20 (TBS-T) supplemented with $5 \%$ nonfat milk, membranes were incubated overnight at $4^{\circ} \mathrm{C}$ with primary antibodies against Bak1 (monoclonal, $1: 250, \mathrm{MBL})$, PARP $(1: 1000, \mathrm{BD})$, Caspase-3 (1:1000, BD), Caspase-9 (1:1000, MBL), Caspase-2 (1:1000, $\mathrm{BD}), \mathrm{BMF}$ (polyclonal, 1:1000, MBL), PUMA (monoclonal, $1: 1000, \mathrm{BD}), \mathrm{Bcl}-2$ (monoclonal, 1:500, BD), PP2A-catalytic $\alpha(1: 1000, \mathrm{BD}), \mathrm{MCL}-1(1: 1000, \mathrm{BD})$, and $\beta$-actin $(1: 1000$, $\mathrm{BD})$. Membranes were then extensively washed with TBS$\mathrm{T}$ and labeled with horseradish peroxidase-conjugated secondary anti-mouse $(1: 1000, \mathrm{GE})$ or anti-rabbit IgG $(1: 2000$, GE). After additional washes with TBS-T, antigen-antibody complexes were visualized with ECL-Prime Kit (GE).

2.9. Transfection of miRNA to Osteosarcoma Cells Followed by Global Gene Expression Analysis. For preparation of RNA samples, MG63 cells were transfected with $25 \mathrm{nM}$ negative control miRNA, miR-100, and miR-125 using DharmaFECT transfection reagents. Cells were harvested at 72 hours after transfection. mRNA expression profiles of miRNA transfected cells were obtained for the global mRNA expression study using DNA microarray as mentioned above.

\section{Results}

We analyzed global miRNA expression in eight frozen diagnostic open biopsy specimens. Clinical and pathological data are summarized in Table 1 . The tumors included in the miRNA expression study were histologically osteoblastic and originated from either the femur or the tibia. Since a previous study has suggested there was a significant correlation between response to chemotherapy and histology [34], we aimed to omit the histological bias in our study by using samples with similar histological and anatomical backgrounds. Patients who had less than $10 \%$ viable tumor cells after chemotherapy were considered good responders, and all others were deemed to be poor responders according to the Huvos grading system. Expression profiling identified six miRNAs expressed at significantly different levels $(p<0.05)$ between good responders and poor responders (Figure 1(a), Supplementary Table 1 in Supplementary Material available online at http://dx.doi.org/10.1155/2016/1390571). Of these, the miRNAs 483-3p, 100, 124, 125b, and 127-3p were expressed more abundantly in poor responders, while miR-887 expression was suppressed. Clinical or pathological factors were not obviously correlated with differential expression. To confirm results, these miRNAs were amplified by qRT-PCR from the same eight samples. This assay confirmed significantly different $(p<0.05)$ expression of miR-125b and miR-100 in poor responders (Figures $1(\mathrm{~b})$ and $1(\mathrm{c})$ ) but not of miRNAs 124, 127-3p, 483-3p, and 887 (Figures $1(\mathrm{~d})-1(\mathrm{~g})$ ). Nevertheless, expression levels measured by qRT-PCR show the same general trends as microarray data.

To investigate these observations further, we measured miRNA expression by qRT-PCR in 20 formalin-fixed paraffin-embedded open biopsy samples of primary osteosarcoma (Supplementary Table 2). The tumor tissues used for validation purposes were heterogeneous; they included osteoblastic, chondroblastic, or fibroblastic tumors, and their site of origin included the tibia, the femur, or the ilium. By exploring such heterogeneous tumor samples, we aimed to demonstrate the versatility of these miRs in predicting responsiveness to neoadjuvant chemotherapy. We also found miR-125b and miR-100 to be expressed at significantly higher levels $(p<0.05)$ in poor responders (Figures $2(a)$ and $2(b)$ ). The receiver operating characteristic curve had areas under the curves 0.909 and 0.899 for miR-125b and miR-100, respectively ( $p<0.05$, Figures 2(c) and 2(d)).

In light of these results, we characterized the functional significance of miR-125b and miR-100 overexpression in osteosarcoma cells. In cell proliferation assays, we observed miR-125b and miR-100 to significantly enhance growth in MNNG/HOS, 143B, and MG63 (Figures 3(a)-3(c)). Moreover, we found that miR-125b promoted cell invasiveness in MNNG/HOS and 143B, while miR-100 enhanced cell invasiveness in all three cell lines (Figures 4(a) and 4(b)). These observations suggested that overexpression of miR-125b and miR-100 had significant effects on tumorigenicity. 


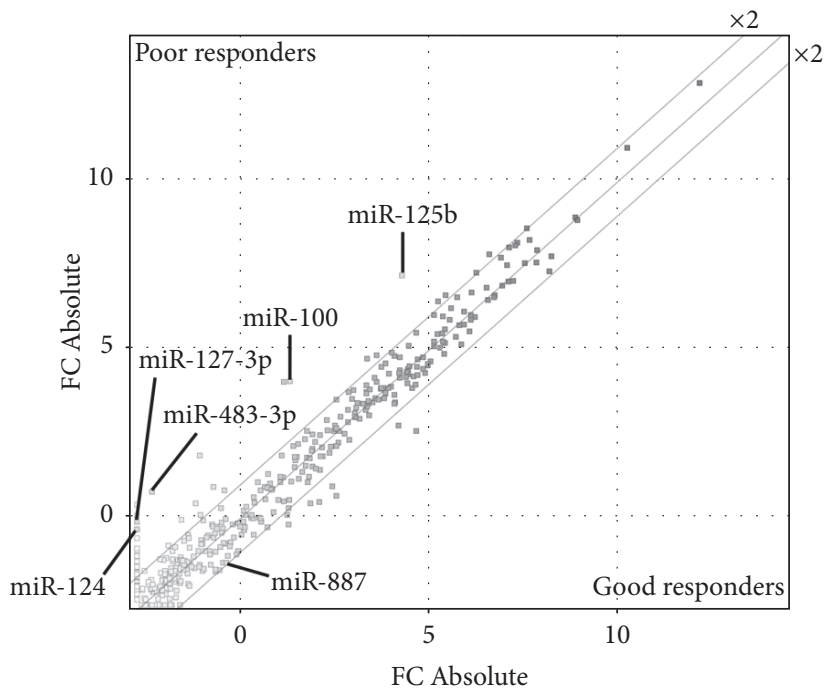

(a)

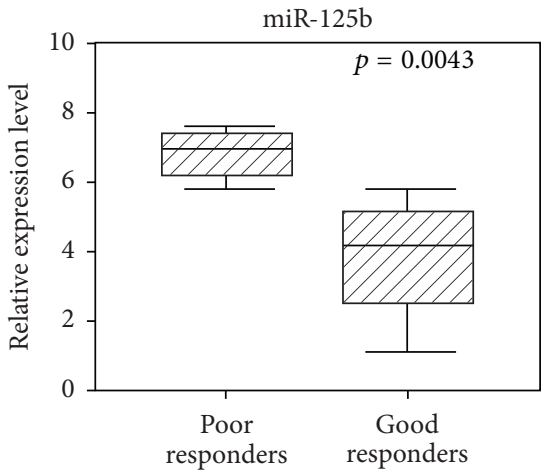

(b)

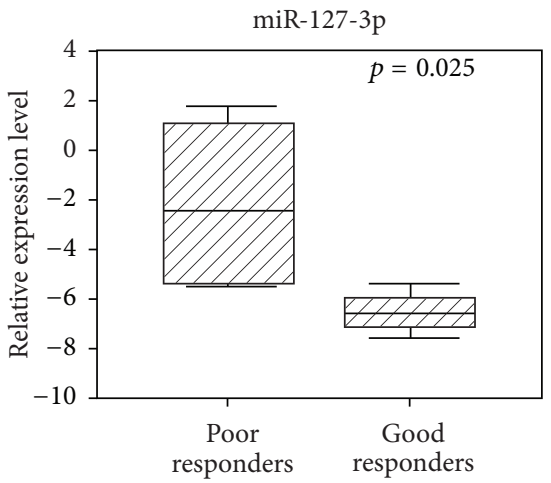

(e)

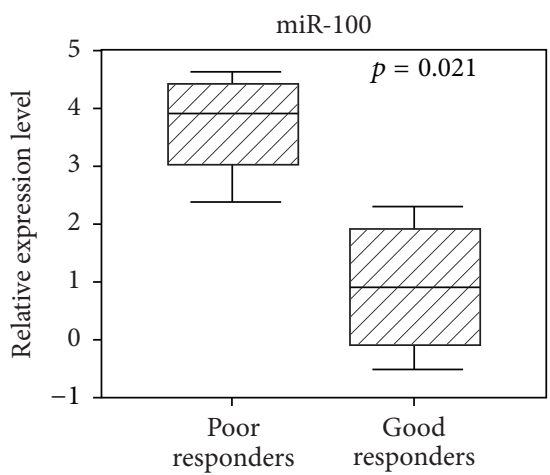

(c)

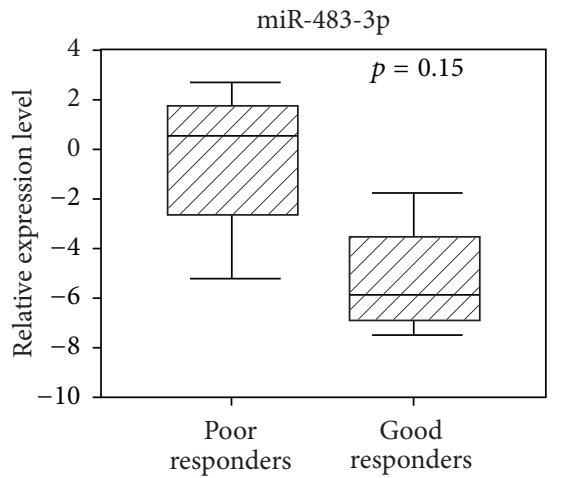

(f)

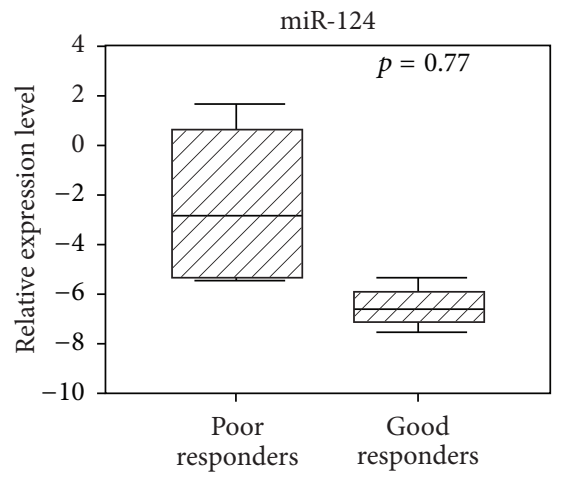

(d)

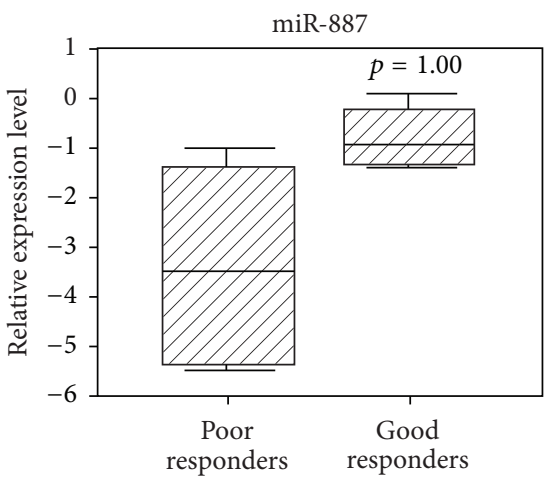

(g)

FIGURE 1: Expression of six miRNAs was significantly different between poor and good responders, as measured by microarray analysis of open biopsy samples (a). qRT-PCR (b-g) of these six miRNAs confirmed that miRNA-125b (b) and miR-100 (c) were expressed at significantly higher levels in chemoresistant patients.

We investigated the impact of miR-125b and miR-100 on the effectiveness of chemotherapy drugs. Overexpression of miR-125b significantly blocked the cytotoxic effects of methotrexate, doxorubicin, and cisplatin in all cells (Supplementary Figure 1, Supplementary Table 3). miR-100 also blocked cytotoxicity, except that of doxorubicin in MNNG/ HOS cells (Supplementary Figure 1, Supplementary Table 3).
Subsequently, we focused on miR-125b because of its ability to block all chemotherapy drugs in all cells examined.

We hypothesized that the known ability of miR-125b to inhibit apoptosis drives tumor progression and resistance to chemotherapy. Indeed, we found that overexpression of miR$125 \mathrm{~b}$ markedly reduced the expression of apoptosis proteins, including p53, Caspase-2, MCL-1, PUMA, and PP2A catalytic 


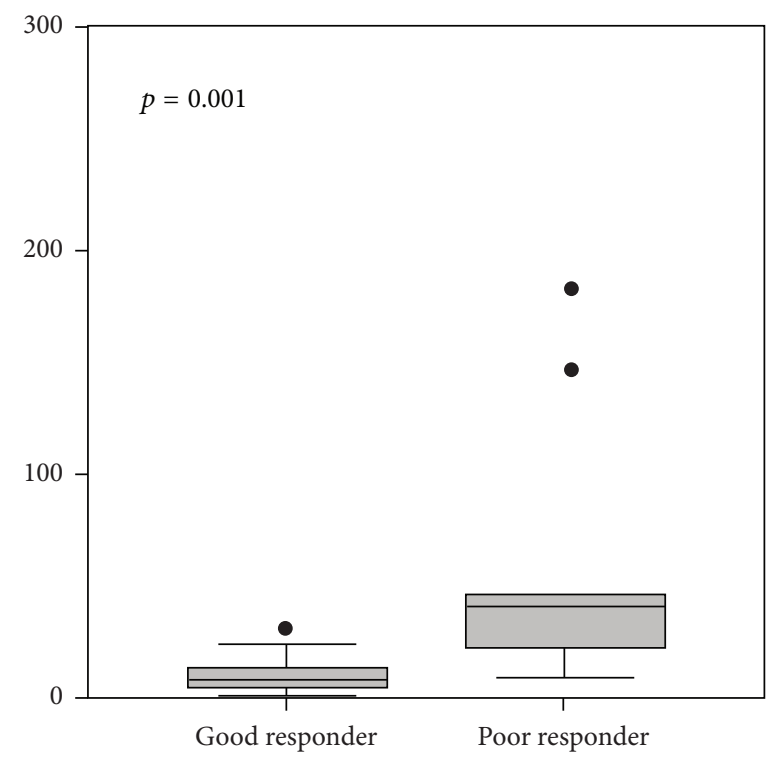

(a)

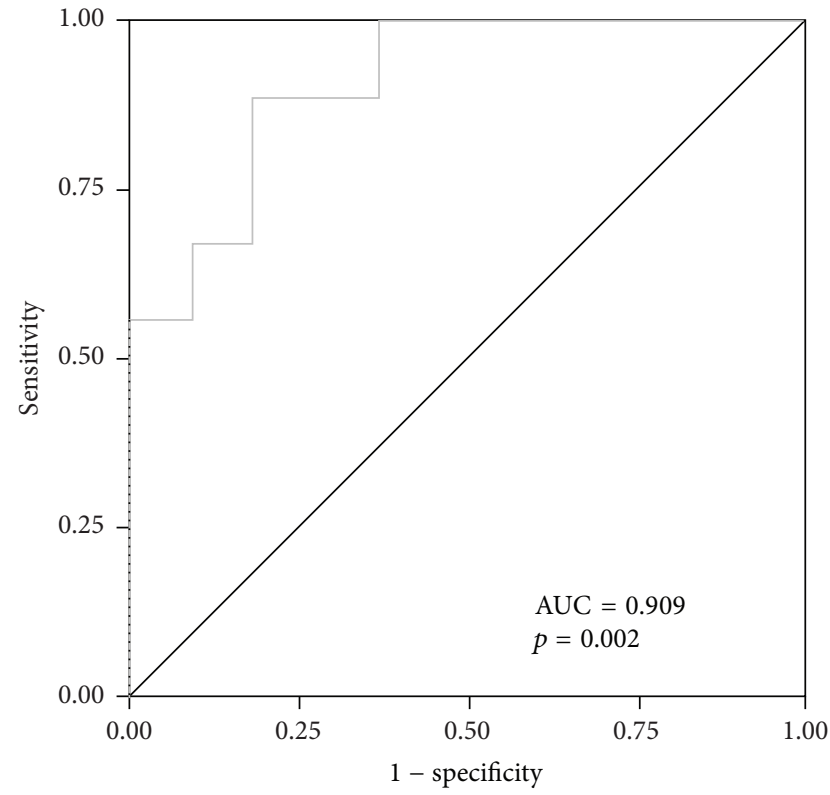

(c)

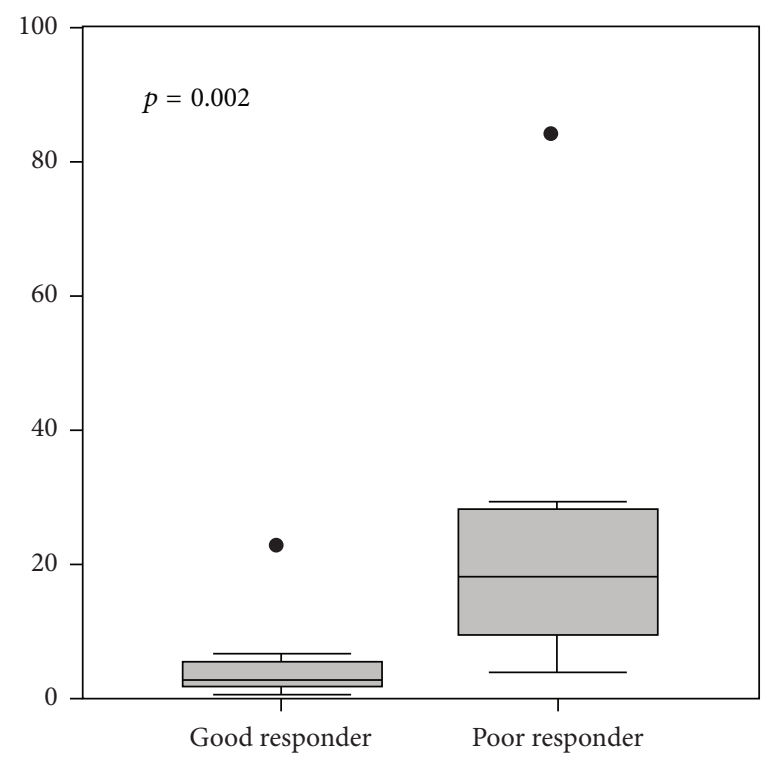

(b)

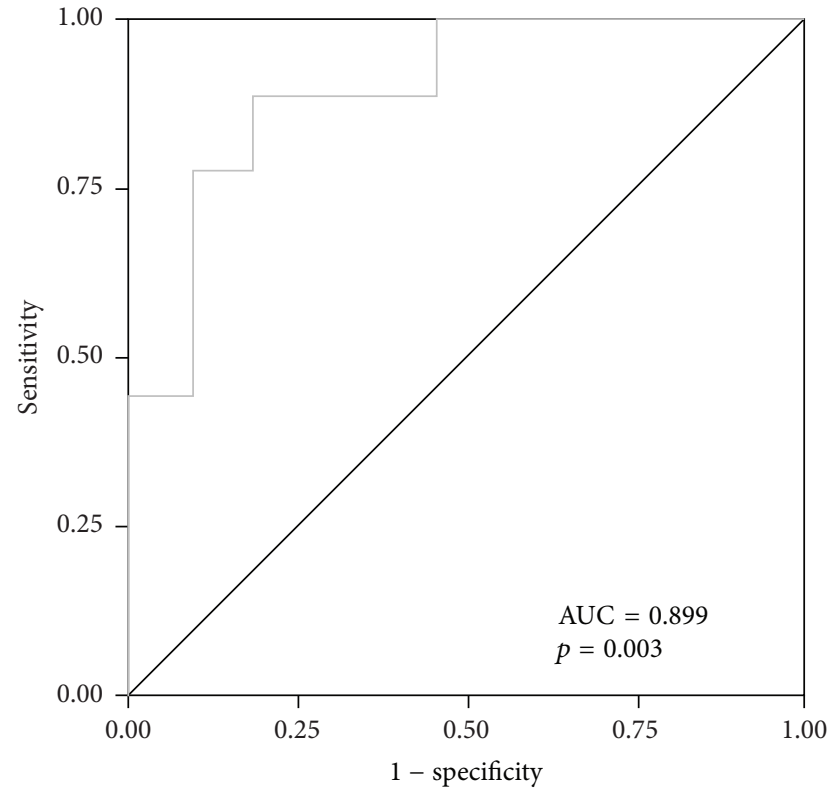

(d)

FIGURE 2: In an independent cohort of patients, expression of miR-125b (a) and miR-100 (b) was higher in patients resistant induction chemotherapy. miR-125b (c) and miR-100 (d) showed significant sensitivity and specificity in receiver operating curves, with area under the curve 0.909 and 0.899 , respectively. The black circles represent the patients' data.

$\alpha$ (Supplementary Figure 2(A)). We also found that miR-125b blocked the ability of methotrexate, doxorubicin, and cisplatin to induce expression of Bak1 and cleaved PARP (Supplementary Figure 2(B)). On the other hand, overexpression of miR-100 resulted in reduced expression of CTDSPL and $\mathrm{Rb}$ (Supplementary Figure 2(C)). These results are consistent with our hypothesis that miR-125b may contribute to drug resistance by inhibiting apoptosis.

To explore the effects of miR-100 and miR-125b, we examined the global expression of mRNA after transfecting miR100 or miR-125b. The transfection of miR-100 and miR-125b induced the differential expression of 35 and 88 genes, respectively (Supplementary Tables 4 and 5). The 16 mRNAs were commonly up- or downregulated by transfection of miR-100 and miR-125b (Table 3).

\section{Discussion}

As osteosarcoma patients have significantly favorable prognosis when they respond well to neoadjuvant treatments, molecular biomarkers predictive of this response may be useful to determine the appropriate course of treatment. 


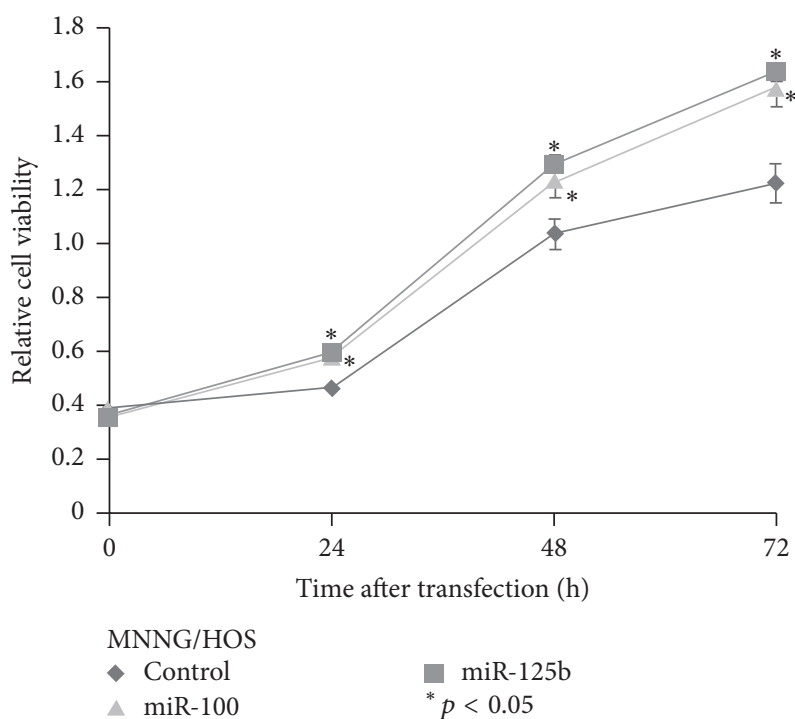

(a)

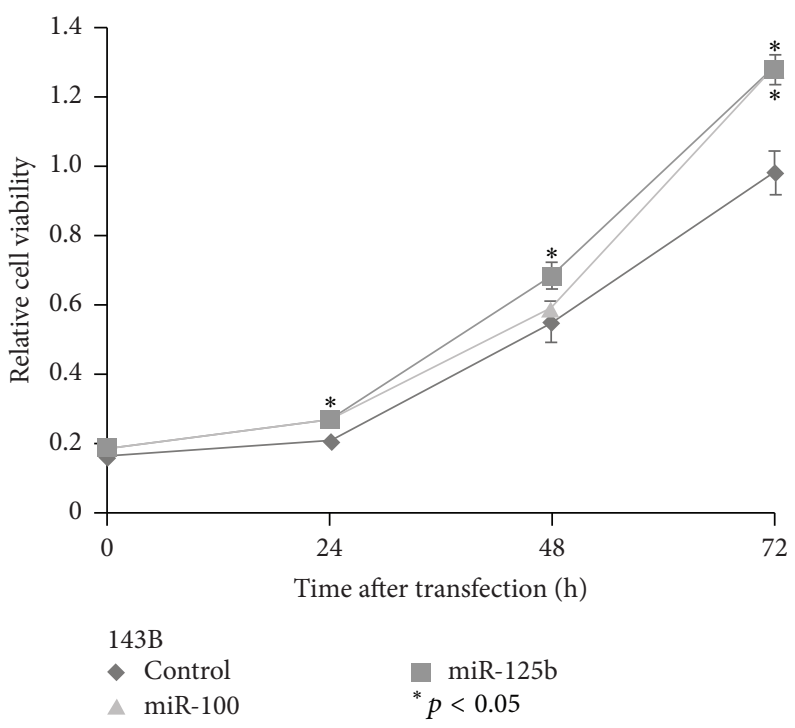

(b)

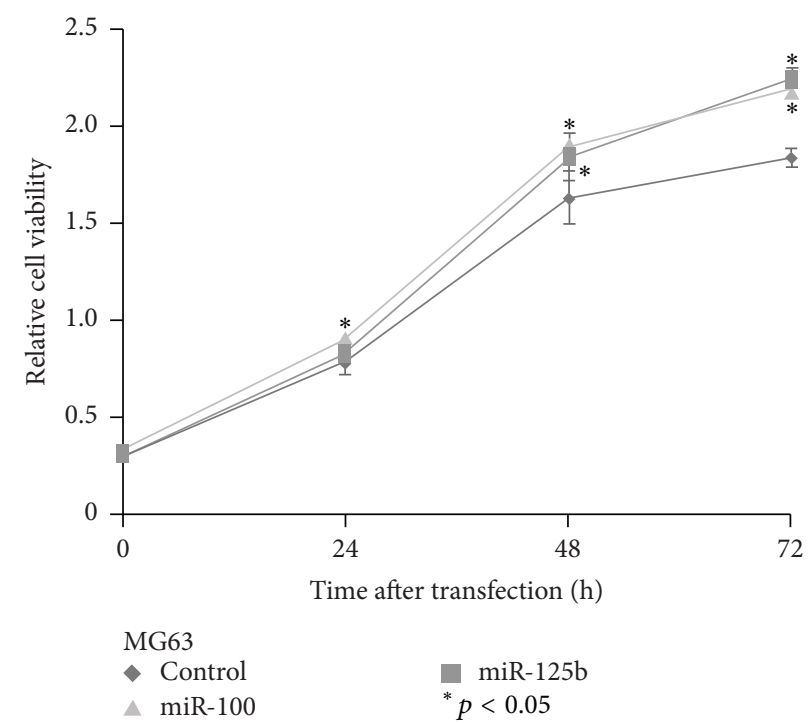

(c)

FIGURE 3: Transfection of miRNA-100 and miR-125b significantly enhanced $(p<0.05)$ proliferation in osteosarcoma cell lines MNNG/HOS (a), 143B (b), and MG63 (c).

Several of such biomarkers have been reported, including P-glycoprotein [15] and PRDX2, which was associated with resistance to neoadjuvant treatments in two independent studies [20, 21]. In contrast, Borys et al. [35] reported that p16 was correlated with a therapeutic response. Although these studies concerning miR-100 and miR-125b appear promising, there is insufficient evidence to support their practical use. Extensive validation studies will be required to establish the predictive utility of these biomarkers prior to application in the clinic.

We now report that abundant expression of miR-100 and $\mathrm{miR}-125 \mathrm{~b}$ in osteosarcoma tissues prior to treatment is predictive of a poor response to neoadjuvant chemotherapy. Notably, expression of miR-100 and miR-125b was found to be lower in osteosarcoma tissues than in adjacent healthy tissues $[36,37]$. As we examined expression of these miRNAs only in tumor tissues, it might be informative to investigate whether expression in adjacent tissues is correlated in some way with clinical and pathological parameters. The superiority of miR100 and miR-125b over other previously reported predictive biomarkers should be noted. The area under the ROC curve for PRDX2 has been reported to be $0.90(p=0.015)[20,21]$. The predictive performances of miR-100 and miR-125b noted here (areas under the ROC curve of approximately 0.9 in each case) are equivalent to that of PRDX2. However, the expression of PRDX2 was measured by western blotting, a methodology that cannot be easily automated. In contrast, the expression levels of miR-100 and miR-125b were measured 

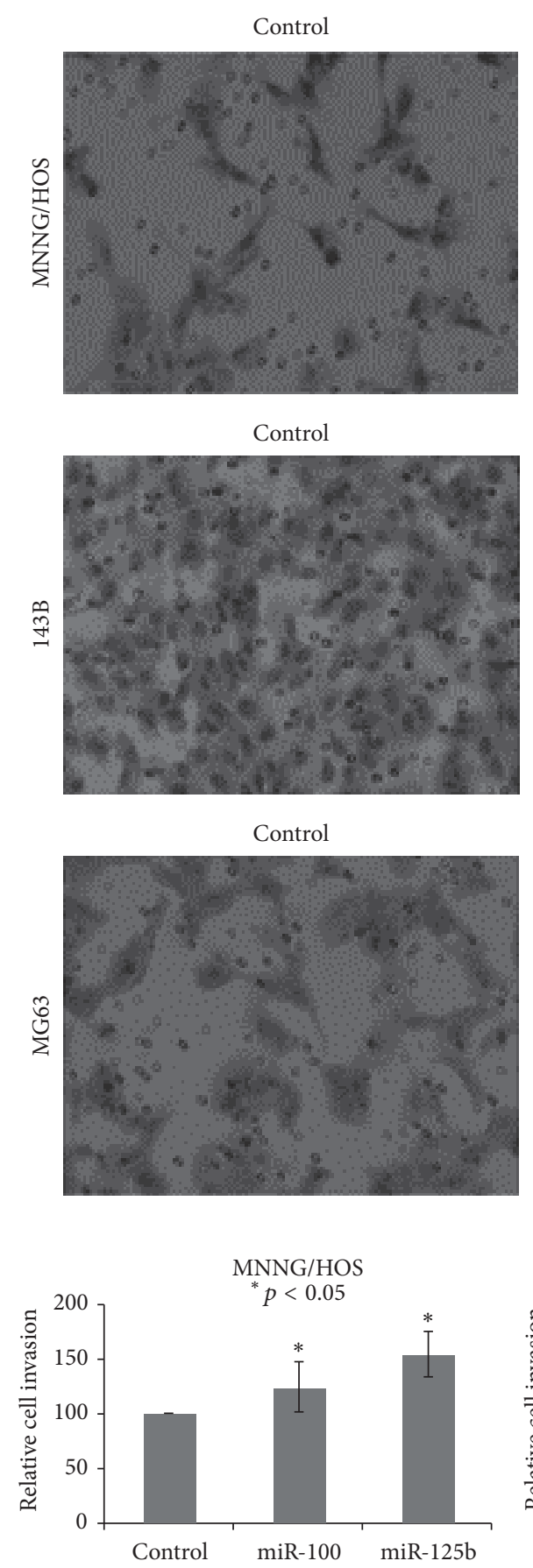

(b)
miR-100

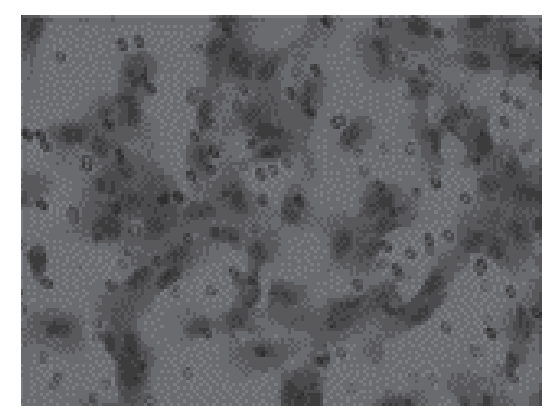

miR-100

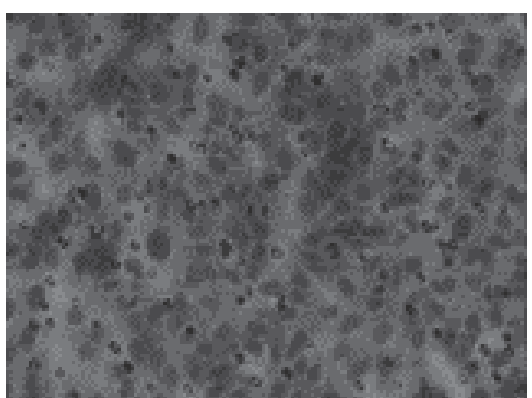

miR-100

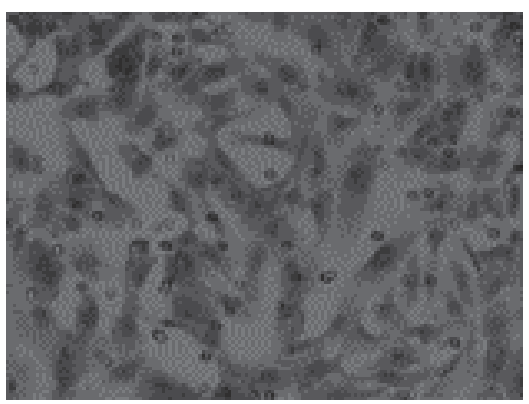

(a)

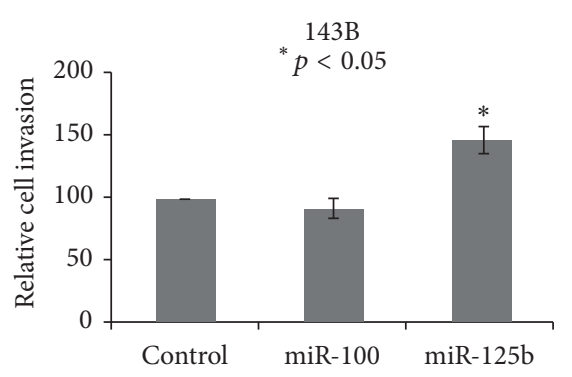

(c)
miR-125b

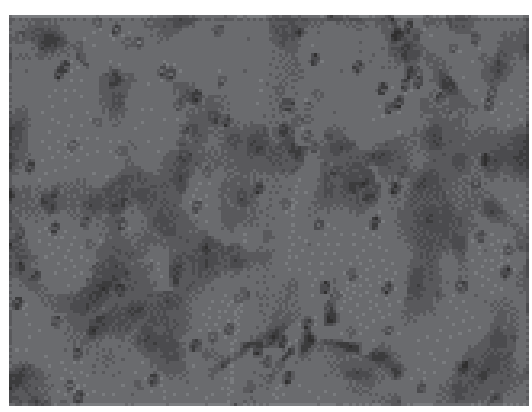

miR-125b

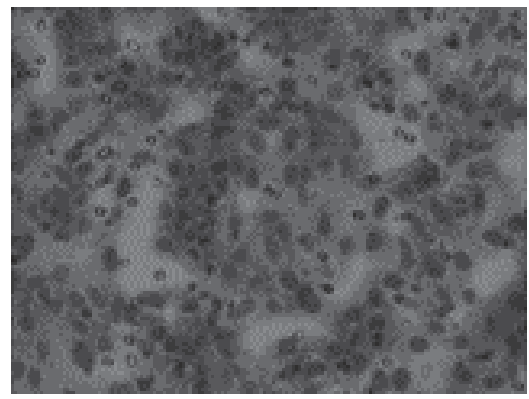

miR-125b
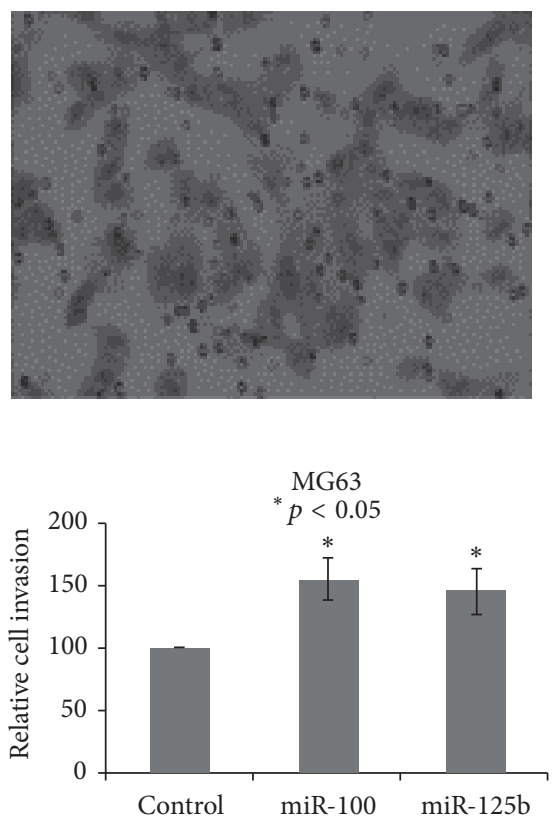

(d)

Figure 4: Transfection of miR-100 and miR-125b enhanced cell invasiveness in MNNG/HOS (a), 143B (b), and MG63 (c) osteosarcoma cell lines.

by qRT-PCR, which can be easily automated. The practical utility of miR-100 and miR-125b should therefore be further explored.

The reported effects of miR-100 and miR-125b on proliferation of osteosarcoma cells are controversial. Huang et al. [36] demonstrated that miR-100 suppresses proliferation of Saos-2 and MG63 cells, an effect reversed by antisense RNA against miR-100. In contrast, we found miR-100 as well as miR-125b to stimulate proliferation in MNNB/HOS, 143B, and MG63 cells (Figure 3). A possible explanation for this discrepancy is the use of different assays to measure cell proliferation [33]. As osteosarcoma is a heterogeneous malignancy, in order to establish the molecular mechanisms of chemoresistance we may need to explore a range of osteosarcoma cell lines.

We found the mRNAs expression which was commonly upregulated by the transfection of miR-100 and miR-125b. 
TABLE 3: Genes commonly regulated by miRNA-100 and miRNA-125b.

\begin{tabular}{lccc}
\hline Accession number & Symbol & Gene title & Biological function \\
\hline Upregulated genes & & & Nucleotide binding \\
NM_006868 & RAB31 & RAB31, member RAS oncogene family & Cation channel activity \\
NM_012241 & SIRT5 & Sirtuin (silent mating type information regulation 2 homolog) 5 & NAD+ ADP-ribosyltransferase activity \\
NM_001142864 & PIEZO1 & Piezo-type mechanosensitive ion channel component 1 & Metal ion binding \\
NM_002383 & MAZ & MYC-associated zinc finger protein & Structural constituent of muscle \\
NM_004997 & MYBPH & Myosin binding protein H & Structural molecule activity \\
NM_000261 & MYOC & Myocilin, trabecular meshwork inducible glucocorticoid response & Chromatin binding \\
NM_014292 & CBX6 & Chromobox homolog 6 & Unknown \\
NM_145267 & C6orf57 & Chromosome 6 open reading frame 57 & Cytokine activity \\
\hline Downregulated genes & & & Calcium ion binding \\
NM_000576 & IL1B & Interleukin 1, beta & Structural molecule activity \\
NM_014510 & PCLO & piccolo (presynaptic cytomatrix protein) & Cytokine activity \\
NM_001017402 & LAMB3 & Laminin, beta 3 & Nucleotide binding \\
NM_004864 & GDF15 & Growth differentiation factor 15 & Structural molecule activity \\
NM_000379 & XDH & Xanthine dehydrogenase & Unknown \\
NM_182507 & KRT80 & Keratin 80 & Unknown \\
NM_213602 & SIGLEC15 & Sialic acid binding Ig-like lectin 15 & Lymphocyte cytosolic protein 2 \\
NM_005565 & LCP2 & &
\end{tabular}

Among them, the expression of sirtuin (silent mating type information regulation 2 homolog 5, SIRT5) was previously associated with the resistance against the in vitro and in vivo treatments with cis-diamminedichloroplatinum, 5fluorouracil, or bleomycin in non-small cell lung cancer [38]. The aberrant regulation of SIRT5 was reported in various types of malignancies. For example, in breast cancer, the expression of SIRT5 had significant relation with tumor location, grade, and expression of estrogen receptor or progesterone receptor [39]. However, the aberrant expression of SIRT5 regulated by miRNAs was not implicated with osteosarcoma until our study. The sirtuin family genes play an important role in the carcinogenesis and cancer progression [40], and it is worth challenging to investigate the regulation and functions of sirtuins in osteosarcomas.

Our next challenge is to establish the clinical utility of miR-100 and miR-125b. As the number of cases in this study was limited, more validation studies may be required before clinical application. Prediction of a poor response to neoadjuvant chemotherapy may prevent the use of time-consuming but ultimately ineffective treatments and thereby prevent patients from experiencing unnecessary side effects. Unfortunately, there are no alternative therapeutic strategies. Nevertheless, immediate surgical resection due to a predicted risk of chemoresistance may improve clinical outcomes for some patients. Identification of chemoresistant patients may also justify their inclusion in clinical trials of novel anticancer agents. Finally, we may be able to identify new therapeutic targets by characterizing the molecular basis of the correlation between miR-100 and miR-125b expression and response to neoadjuvant chemotherapy.

The global expression of miRNAs and especially the expression level of miR-100 and miR-125b in metastatic tumors is intriguing. Recently, Berlanga et al. compared primary osteosarcomas with lung metastatic osteosarcomas and identified twenty-six miRNAs with significantly different expression between the two [41]. Although miR-100 and miR-125b were not identified in their study, it is worth examining their expression in paired primary and metastatic tumor tissues in patients having different responses to chemotherapeutic treatment. The correlation between the miR-100 and miR$125 \mathrm{~b}$ expression levels and tumor recurrence is also worth exploring. Sanchez-Diaz et al. have previously reported the identity of miRNAs associated with tumor recurrence in pediatric osteosarcoma [42]. Probably because they focused on pediatric osteosarcoma, miR-100 and miR-125b were not identified as being associated with tumor recurrence. As the response to neoadjuvant chemotherapy is associated with a favorable prognosis, the expression of miR-100 and miR-125b may be associated with poor prognosis in osteosarcoma. This hypothesis should be addressed in a future study.

In summary, expression of miR-100 and miR-125b in pretreatment of osteosarcoma is significantly correlated with poor chemotherapy response and is a promising biomarker to guide treatment decisions.

\section{Disclosure}

Present address of Daisuke Kubota is Department of Orthopaedic Surgery, Juntendo University School of Medicine, Tokyo, Japan. Present address of Nobuyoshi Kosaka is Department of Zoology, University of Oxford, Oxford, UK. Present address of Tomohiro Fujiwara is Department of Orthopaedic Surgery, Okayama University Graduate School of Medicine, Dentistry, and Pharmaceutical Sciences, Okayama, Japan. 


\section{Competing Interests}

The authors declare that they have no conflict of interests.

\section{Acknowledgments}

This research was supported by Practical Research for Innovative Cancer Control (15ck0106089h0002 and 15ck0106089h0003) from Japan Agency for Medical Research and Development, by the National Cancer Center Development Fund (26-A-3), and by Grant-in-Aid for Scientific Research 25871161.

\section{References}

[1] C. P. Gibbs, K. Weber, and M. T. Scarborough, "Malignant bone tumors," Journal of Bone and Joint Surgery-Series A, vol. 83, no. 11, pp. 1728-1745, 2001.

[2] P. A. Meyers and R. Gorlick, "Osteosarcoma," The Pediatric Clinics of North America, vol. 44, no. 4, pp. 973-989, 1997.

[3] A. J. Provisor, L. J. Ettinger, J. B. Nachman et al., "Treatment of nonmetastatic osteosarcoma of the extremity with preoperative and postoperative chemotherapy: a report from the Children's Cancer Group," Journal of Clinical Oncology, vol. 15, no. 1, pp. 76-84, 1997.

[4] G. Rosen, "Preoperative (neoadjuvant) chemotherapy for osteogenic sarcoma: a ten year experience," Orthopedics, vol. 8, no. 5, pp. 659-664, 1985.

[5] G. Rosen, R. C. Marcove, A. G. Huvos et al., "Primary osteogenic sarcoma: eight-year experience with adjuvant chemotherapy," Journal of Cancer Research and Clinical Oncology, vol. 106, no. 1, pp. 55-67, 1983.

[6] G. Rosen, B. Caparros, S. Groshen et al., "Primary osteogenic sarcoma of the femur: a model for the use of preoperative chemotherapy in high risk malignant tumors," Cancer Investigation, vol. 2, no. 3, pp. 181-192, 1984.

[7] G. Bacci, M. Avella, D. P. A. Brach et al., "Neoadjuvant chemotherapy for osteosarcoma of the extremities. Good response of the primary tumor after preoperative chemotherapy with highdose methotrexate followed by cisplatinum and adriamycin. Preliminary results," Chemioterapia, vol. 7, no. 2, pp. 138-142, 1988.

[8] K. Winkler, G. Beron, G. Delling et al., "Neoadjuvant chemotherapy of osteosarcoma: results of a randomized cooperative trial (COSS-82) with salvage chemotherapy based on histological tumor response," Journal of Clinical Oncology, vol. 6, no. 2, pp. 329-337, 1988.

[9] A. M. Davis, R. S. Bell, and P. J. Goodwin, "Prognostic factors in osteosarcoma: a critical review," Journal of Clinical Oncology, vol. 12, no. 2, pp. 423-431, 1994.

[10] S. Weeden, R. J. Grimer, S. R. Cannon, A. H. M. Taminiau, and B. M. Uscinska, "The effect of local recurrence on survival in resected osteosarcoma," European Journal of Cancer, vol. 37, no. 1, pp. 39-46, 2001.

[11] G. Bacci, A. Longhi, M. Versari, M. Mercuri, A. Briccoli, and P. Picci, "Prognostic factors for osteosarcoma of the extremity treated with neoadjuvant chemotherapy: 15-year experience in 789 patients treated at a single institution," Cancer, vol. 106, no. 5, pp. 1154-1161, 2006.

[12] S. S. Bielack, B. Kempf-Bielack, G. Delling et al., "Prognostic factors in high-grade osteosarcoma of the extremities or trunk. An analysis of 1702 patients treated on Cooperative Osteosarcoma Study Group protocols," Journal of Clinical Oncology, vol. 20, no. 3, pp. 776-790, 2002.

[13] D. B. Glasser, J. M. Lane, A. G. Huvos, R. C. Marcove, and G. Rosen, "Survival, prognosis, and therapeutic response in osteogenic sarcoma. The memorial hospital experience," Cancer, vol. 69, no. 3, pp. 698-708, 1992.

[14] T. Akatsuka, T. Wada, Y. Kokai et al., "ErbB2 expression is correlated with increased survival of patients with osteosarcoma," Cancer, vol. 94, no. 5, pp. 1397-1404, 2002.

[15] N. Baldini, K. Scotlandi, G. Barbanti-Bròdano et al., "Expression of P-glycoprotein in high-grade osteosarcomas in relation to clinical outcome," The New England Journal of Medicine, vol. 333, no. 21, pp. 1380-1385, 1995.

[16] G. Rosen, B. Caparros, A. G. Huvos et al., "Preoperative chemotherapy for osteogenic sarcoma: selection of postoperative adjuvant chemotherapy based on the response of the primary tumor to preoperative chemotherapy," Cancer, vol. 49, no. 6, pp. 1221-1230, 1982.

[17] M. J. Klein and G. P. Siegal, "Osteosarcoma: anatomic and histologic variants," American Journal of Clinical Pathology, vol. 125, no. 4, pp. 555-581, 2006.

[18] M. L. Kuijjer, P. C. W. Hogendoorn, and A.-M. Cleton-Jansen, "Genome-wide analyses on high-grade osteosarcoma: making sense of a genomically most unstable tumor," International Journal of Cancer, vol. 133, no. 11, pp. 2512-2521, 2013.

[19] A. K. Boehm, J. R. Neff, J. A. Squire, J. Bayani, M. Nelson, and J. A. Bridge, "Cytogenetic findings in 36 osteosarcoma specimens and a review of the literature," Pediatric Pathology and Molecular Medicine, vol. 19, no. 5, pp. 359-376, 2000.

[20] K. Kikuta, N. Tochigi, S. Saito et al., "Peroxiredoxin 2 as a chemotherapy responsiveness biomarker candidate in osteosarcoma revealed by proteomics," Proteomics-Clinical Applications, vol. 4, no. 5, pp. 560-567, 2010.

[21] D. Kubota, K. Mukaihara, A. Yoshida, H. Tsuda, A. Kawai, and T. Kondo, "Proteomics study of open biopsy samples identifies peroxiredoxin 2 as a predictive biomarker of response to induction chemotherapy in osteosarcoma," Journal of Proteomics, vol. 91, pp. 393-404, 2013.

[22] D. P. Bartel, "MicroRNAs: genomics, biogenesis, mechanism, and function," Cell, vol. 116, no. 2, pp. 281-297, 2004.

[23] B. P. Lewis, C. B. Burge, and D. P. Bartel, "Conserved seed pairing, often flanked by adenosines, indicates that thousands of human genes are microRNA targets," Cell, vol. 120, no. 1, pp. 1520, 2005.

[24] L. P. Lim, N. C. Lau, P. Garrett-Engele et al., "Microarray analysis shows that some microRNAs downregulate large numbers of target mRNAs," Nature, vol. 433, no. 7027, pp. 769-773, 2005.

[25] R. R. Lulla, F. F. Costa, J. M. Bischof et al., "Identification of differentially expressed microRNAs in osteosarcoma," Sarcoma, vol. 2011, Article ID 732690, 6 pages, 2011.

[26] G. Maire, J. W. Martin, M. Yoshimoto, S. Chilton-MacNeill, M. Zielenska, and J. A. Squire, "Analysis of miRNA-gene expression-genomic profiles reveals complex mechanisms of microRNA deregulation in osteosarcoma," Cancer Genetics, vol. 204, no. 3, pp. 138-146, 2011.

[27] E. Kobayashi, R. Satow, M. Ono et al., "MicroRNA expression and functional profiles of osteosarcoma," Oncology, vol. 86, no. 2, pp. 94-103, 2014.

[28] K. B. Jones, Z. Salah, S. Del Mare et al., "miRNA signatures associate with pathogenesis and progression of osteosarcoma," Cancer Research, vol. 72, no. 7, pp. 1865-1877, 2012. 
[29] M. B. Mintz, R. Sowers, K. M. Brown et al., "An expression signature classifies chemotherapy-resistant pediatric osteosarcoma," Cancer Research, vol. 65, no. 5, pp. 1748-1754, 2005.

[30] A. Gougelet, D. Pissaloux, A. Besse et al., "Micro-RNA profiles in osteosarcoma as a predictive tool for ifosfamide response," International Journal of Cancer, vol. 129, no. 3, pp. 680-690, 2011.

[31] A. D. Kelly, B. Haibe-Kains, K. A. Janeway et al., "MicroRNA paraffin-based studies in osteosarcoma reveal reproducible independent prognostic profiles at 14q32," Genome Medicine, vol. 5, no. 1, article 2, 2013.

[32] Y. Iwamoto, K. Tanaka, K. Isu et al., "Multiinstitutional phase II study of neoadjuvant chemotherapy for osteosarcoma (NECO study) in Japan: NECO-93J and NECO-95J," Journal of Orthopaedic Science, vol. 14, no. 4, pp. 397-404, 2009.

[33] M. Ishiyama, Y. Miyazono, K. Sasamoto, Y. Ohkura, and K. Ueno, "A highly water-soluble disulfonated tetrazolium salt as a chromogenic indicator for $\mathrm{NADH}$ as well as cell viability," Talanta, vol. 44, no. 7, pp. 1299-1305, 1997.

[34] G. Bacci, S. Ferrari, N. Delepine et al., "Predictive factors of histologic response to primary chemotherapy in osteosarcoma of the extremity: study of 272 patients preoperatively treated with high-dose methotrexate, doxorubicin, and cisplatin," Journal of Clinical Oncology, vol. 16, no. 2, pp. 658-663, 1998.

[35] D. Borys, R. J. Canter, B. Hoch et al., "P16 expression predicts necrotic response among patients with osteosarcoma receiving neoadjuvant chemotherapy," Human Pathology, vol. 43, no. 11, pp. 1948-1954, 2012.

[36] J. Huang, K. Gao, J. Lin, and Q. Wang, "MicroRNA-100 inhibits osteosarcoma cell proliferation by targeting Cyr61," Tumor Biology, vol. 35, no. 2, pp. 1095-1100, 2014.

[37] L.-H. Liu, H. Li, J.-P. Li et al., "miR-125b suppresses the proliferation and migration of osteosarcoma cells through downregulation of STAT3," Biochemical and Biophysical Research Communications, vol. 416, no. 1-2, pp. 31-38, 2011.

[38] W. Lu, Y. Zuo, Y. Feng, and M. Zhang, "SIRT5 facilitates cancer cell growth and drug resistance in non-small cell lung cancer," Tumor Biology, vol. 35, no. 11, pp. 10699-10705, 2014.

[39] M. Igci, M. E. Kalender, E. Borazan et al., "High-throughput screening of Sirtuin family of genes in breast cancer," Gene, vol. 586, no. 1, pp. 123-128, 2016.

[40] J. George and N. Ahmad, "Mitochondrial sirtuins in cancer: emerging roles and therapeutic potential," Cancer Research, vol. 76, no. 9, pp. 2500-2506, 2016.

[41] P. Berlanga, L. Muñoz, M. Piqueras et al., "miR-200c and phospho-AKT as prognostic factors and mediators of osteosarcoma progression and lung metastasis," Molecular Oncology, vol. 10, no. 7, pp. 1043-1053, 2016.

[42] P. C. Sanchez-Diaz, T.-H. Hsiao, Y. Zou et al., "In silico functional analyses and discovery of survival-associated microRNA signatures in pediatric osteosarcoma," Oncoscience, vol. 1, no. 9, pp. 599-608, 2014. 


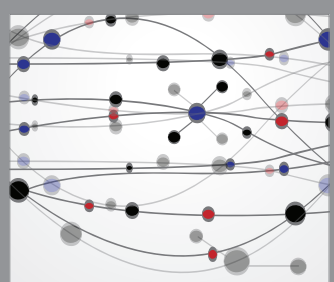

The Scientific World Journal
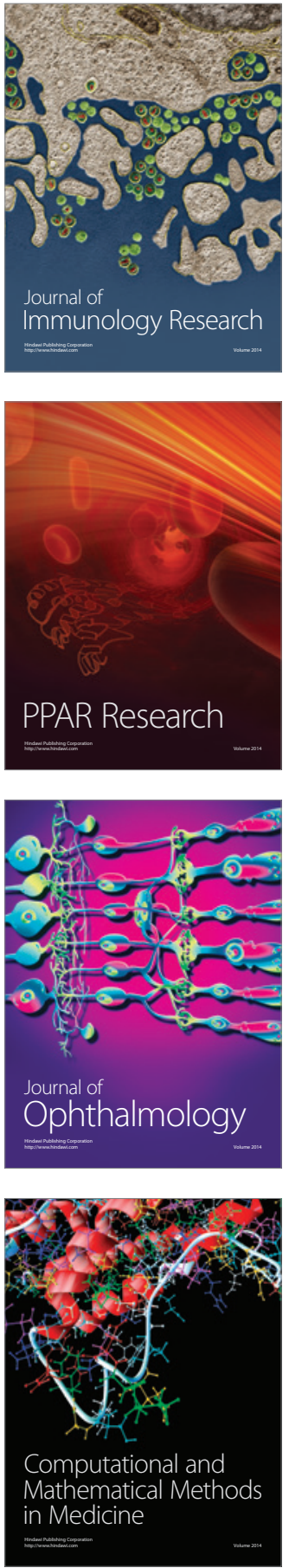

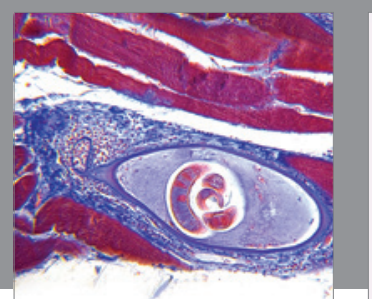

Gastroenterology Research and Practice

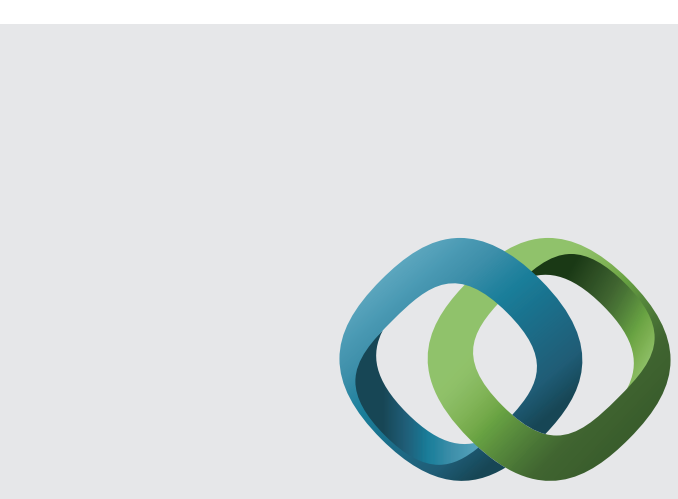

\section{Hindawi}

Submit your manuscripts at

http://www.hindawi.com
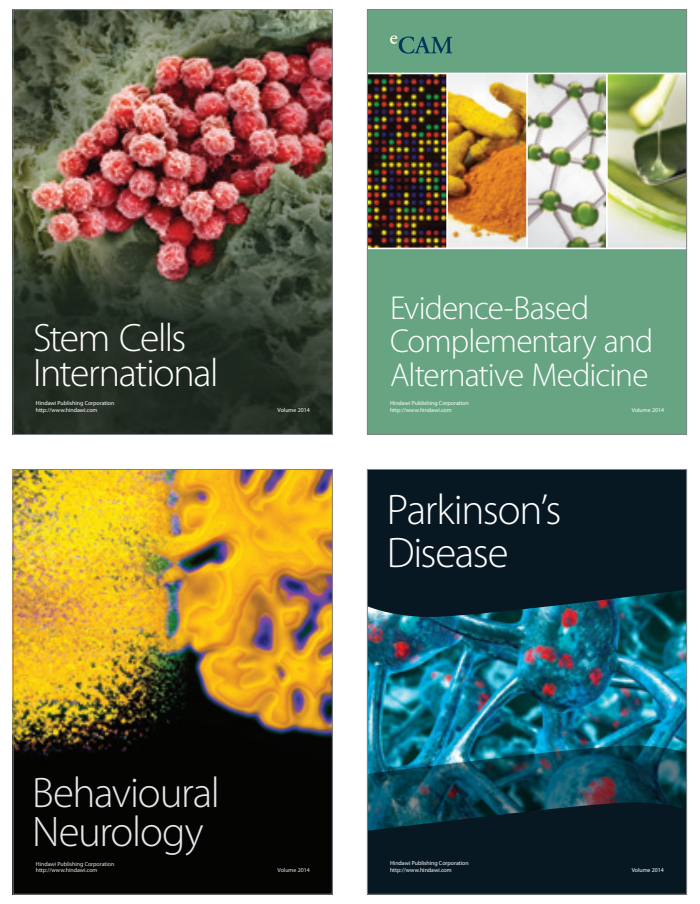
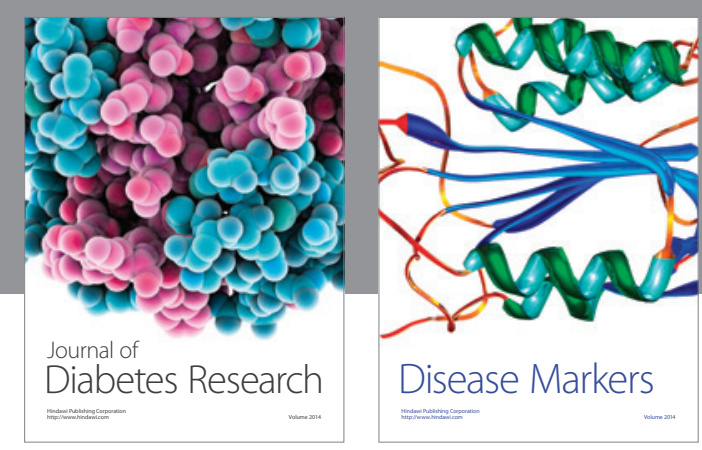

Disease Markers
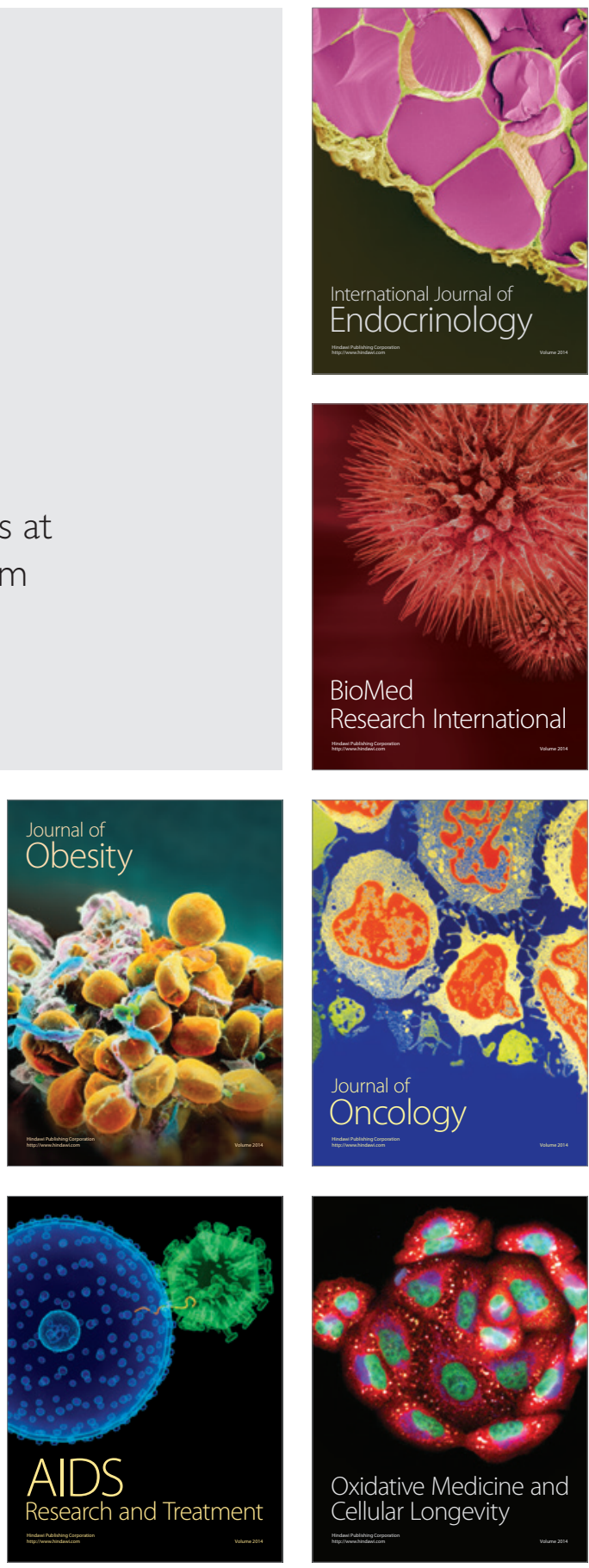